\title{
Normale und pathologische Histologie des Unterhautfettgewebes.
}

\author{
Von
}

\author{
Dr. Louis Heitzmann, \\ Arzt am deutschen Dispensary in New-York. \\ (Mit 6 Abbildungen im Texte.)
}

Eine längst bekannte Thatsache ist, dass von der Lederhaut Züge von fibrösem Bindegewebe in die Tiefe laufen, um sich in die unterliegenden Fascien und Aponeurosen einzusenken. Diese Bündel verlaufen, gleichwie die Bündel der Lederhaut selbst, in schiefer Richtung. Die Räume zwischen diesen Bündeln enthalten das subcutane Fettgewebe, welches selbst bei hochgradig abgemagerten Individuen nie gänzlich fehlt. Man hat die durch gröbere Bindegewebszüge abgegrenzten Fettmassen als Fettläppchen oder Fetträubchen bezeichnet, welche wieder durch zartere Bindegewebszüge in kleinere Läppchen abgetheilt werden. Die allgemeine Form eines Fettläppchens des Unterhautgewebes ist eiue rhombische, entsprechend der Anordnung der begrenzenden Bindegewebsbündel. Jedes Fettläppchen ist mit Fettkugeln erfüllt und von einem überraschenden Reichthum an capillaren Blutgefässen.

Fast alle Histologen beschreiben die Fettkugeln als "Fettzellen" mit einem zähflüssigen, stark lichtbrechenden gelblichen Inhalte, begrenzt von einer dünnen Kapsel, der sogenannten Zellwand, und mit einem meistens excentrisch gelegenen oder wandständigen Kerne versehen. Es gibt Histologen, die die Fettkugel als das Ideal einer Zelle betrachten, 
indem hier alle Bestandtheile einer Zelle, der zähflüssige Inhalt, der Kern und die Zellwand vorhanden sind; dennoch beruht diese Auffassung auf emem Irrthum, wie zuerst von Carl Heitzmann') nachgewiesen wurde.

Betrachten wir das subcutane Fettgewebe in Präparaten, welche nach der herrschenden Methode aus Alkoholobjecten geschnitten, mit Terpentin behandelt und in Canadabalsam montirt wurden, so erkennen wir leere runde oder rundliche Hohlräume, den früheren Fettkugeln entsprechend. Jeder Hohlraum ist von einer überaus zarten Kapsel umgeben, und am äusseren Saume der Kapsel sehen wir einen mit Carmin leicht färbbaren oblongen oder spindelförmigen Kern. Bisweilen liegt der Kern anscheinend innerhalb des Hohlraumes, nahe der Kapsel. Durch genaues Einstellen kann man sich jedesmal überzeugen, dass ein solcher Kern einer dicht oberhalb oder unterhalb liegenden Fettkugel, respective der Kapsel einer solchen Fettkugel angehört. Die Fettkugeln liegen bisweilen dicht beisammen, so zwar, dass sich die benachbarten Kapseln berühren. Viel häufiger aber treffen wir zwischen den einzelnen Fettkugeln ein überaus zartes fibrilläres Bindegewebe, gleichfalls Kerne führend. Zwischen den durch die Kugelform bedingten Interstitien der Fettkugeln bleiben stets grössere Massen solchen zart fibrillären Bindegewebes sichtbar und der oblonge Kern der Kapsel ist stets entsprechend einem solchen breiteren Zwischenraum zu finden. Diese Interstitien enthalten zahlreiche gewundene capillare Blutgefässe, die am injicirten Präparat besonders deutlich hervortreten. (Siehe Fig. 1.)

Aus diesen Thatsachen schliesst der genannte Autor, dass 1. die Fettkugel keine Zelle sei im Sinne der Histologen; 2. dass der Kern nicht der Fettkugel selbst angehört, sondern der umbüllenden Kapsel; 3. dass das Fettgewebe eine Abart myxomatösen Gewebes sei, gebildet von einem Netzwerke zarten fibrösen Bindegewebes, dessen Maschenräume anstatt mit myxomatöser Grundsubstanz, mit halbflüssigem Fette gefüllt sind. In dieser Auffassung wäre die sogenannte Zellwand nichts weiter als eine dünne Lage stark verdichteter, sogenannter elastischer Grundsubstanz, ähnlich jenen Schichten elastischer

1) Mikroskopische Morphologie. 1883. 
Grundsubstanz, welche an den Grenzen der Territorien der Knorpel- und Knochenkörperchen schon längst bekannt sind. Diese Auffassung des Fettgewebes findet sowohl in der Ent-

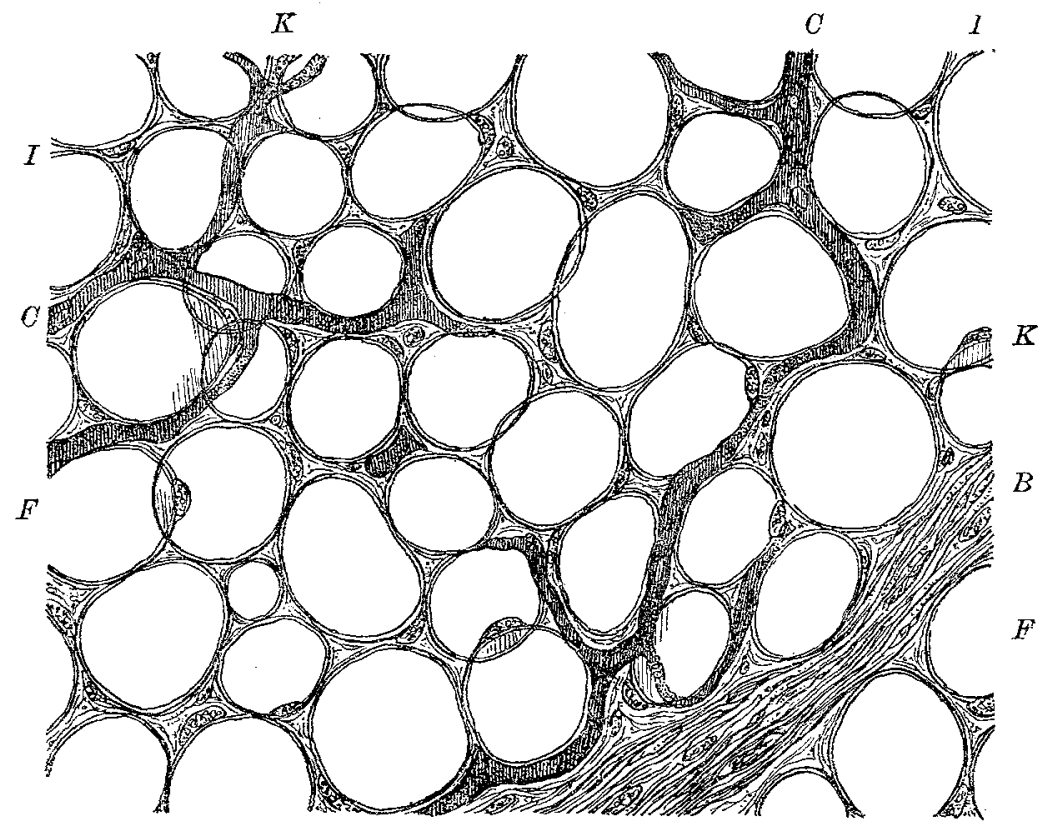

Fig. I. Aus dem Fettgewebe der Gesichtshaut eines neugeborenen Kindes. Das Fett mittelst Terpentin ausgelöst. Canada-Balsam Präparat. Vergr. $=600$.

$B$ Bündel von fibrösem Bindegewebe, zwischen den Fettläppchen. F $F$ Leere Fettkugeln von dünnen Kapseln umgeben. $K K$ Kerne der Kapsel. $I I$ Interstitielle Bindegewebe, gleichfalls kernartig. $C C$ Injicirte capillare Blutgefässe.

wickelungsgeschichte der Fettkugeln, wie auch in Präparaten von rasch abgemagerten Individuen Bestätigung. Wenn E. Klein in London das Fettgewebe eine Abart von Lymphgewebe nennt, so ist das insoferne richtig, als sowohl das Lymph- wie das Fettgewebe Varietäten des myxomatösen Gewebes sind.

Mittelst Reagentien können wir die eigentliche Structur der Fettkugeln nicht aufklären. Terpentin löst zwar das Fett, aber auch alles, was mit Fett infiltrirt war. Carmin und Goldchlorid lässt die Fettkugeln ungefärbt. Osmiumsäure färbt das 
Fett schwarz und ist nur werthvoll zum Sichtbarmachen kleiner Fettkügelchen innerhalb des Protoplasmas, wo Fett eben im Begriffe ist sich zu bilden, insbesondere in den fettbildenden Epithelien der Talgdriisen und der Brustdrüse in den ersten Tagen nach erfolgter Entbindung, in den sogenannten Colostrumkörperchen. Die von uns fast ausschliesslich benutzte Methode der Untersuchung in Chromsäure gehärteter und in Glycerin montirter Präparate lässt beim Studium der Fettkugeln gleichfalls im Stiche. Glycerin löst bekanntlich das Fett sehr allmälig und erhalten wir dadurch Fettkugeln von grobgranulirtem oder vacuolirtem Aussehen, welcher Umstand in die eigentliche Structur der Fettkugeln keinẹn Einblick gewährt. (Siehe Fig. 2.)

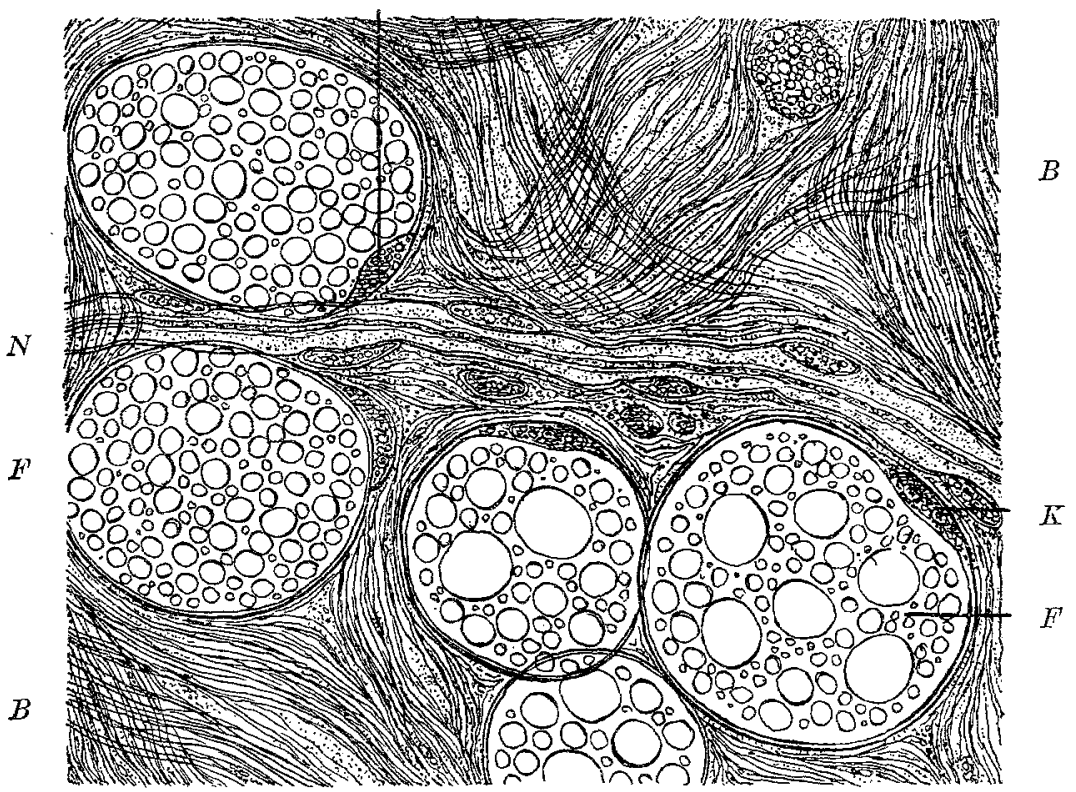

Fig. 2. Fettkugeln aus dem Brustdrüsengewebe. In Chromsäure gehärtet und in Glycerin aufbewahrt. Vergr. $=600$.

$F F$ Reichlich vacuolirte Fettkugeln. $K K$ Kerne der Fettizugelkapseln. $N$ Markhältiger Nerv, das Myelin ausgesickert. $M$ Sogenannte Mastzelle. $B B$ Fibröses Bindegewebe. 
Das abgebildete Präparat stammt aus der Brustdrüse eines Weibes, das wiederholt Kinder geboren hatte. Wir wissen durch die Untersuchungen von C. Langer, dass die jungfräuliche Brustdrüse nur wenig Fett enthält. In der Schwangerschaft hingegen, insbesondere in dem Schrumpfungsprocesse nach beendeter Lactation wird das Fettgewebe der Brustdrüse stark vermehrt und bleibt es bis in das hohe Alter. In der Brustdrüse ist das Fettgewebe ohne Regelmässigkeit mit dem fibrösen Bindegewebe gemengt. Wir treffen Fettkugeln in wechselnder Zahl zwischen dem Drüsengewebe, längs der Blutgefässe und der markhaltigen Nerven, in der Regel in Gestalt vereinzelter Fettkugeln oder Gruppen von solchen. Die typische Anordnung zu Läppchen, wie im übrigen subcutanen Bindegewebe, lässt sich hier nicht nachweisen. Wir erkennen um jede Fettkugel die stark lichtbrechende Kapsel und dicht ausserhalb der Kapsel je einen oblongen oder spindelförmigen Kern. Dieser Umstand spricht zu Gunsten der Auffassung Flemming's, dass die Fettkugeln nicht nothwendigerweise ein Gewebe sui generis bilden müssen, sondern mit fibrösem Bindegewebe gemengt auftreten können.

Der Bau der Fettkugeln wird erst aufgeklärt, wenn wir uns an das Studium der Haut von stark abgemagerten Individuen machen, wie das schon von C. Toldt, ${ }^{1}$ ) $\mathrm{Czajewicz},{ }^{2}$ ) Flemming $g^{3}$ ) u. A. geschehen ist. Die Bilder fallen sehr verschieden aus, je nachdem die Abmagerung eine rasche oder eine allmälige war. Wie schon Toldt richtig bemerkt hat, sind die Veränderungen bei den Fettkugeln durchaus nịcht gleichförmig, selbst nicht bei hochgradiger Abmagerung. Manche Läppchen des Unterhautgewebes können bei rascher Abmagerung unverändert bleiben; andere Läppchen zeigen mehr oder weniger vorgeschrittene Veränderungen. Auch kommt es vor, dass innerhalb eines Läppchens manche Kugeln unverändert geblieben sind, andere hingegen ihr Fett theilweise oder ganz verloren haben und Bilder aufweisen, welche ich im Folgenden beschreiben werde.

1) C. Toldt. Silzungsber, der k. Akad. der Wissensch. Bd.62.1870.

$\left.{ }^{2}\right) \mathrm{Czajewicz} \mathrm{Reichert} \mathrm{u.} \mathrm{du} \mathrm{Brit} \mathrm{Archiv.} 1866$.

3) Flemming Archiv f. mikroskop. Anatomie. 1871. Arehiv f. Dormatol. u. Syphil. Band XXXIr. 
Bei rapider Abzehrung treffen wir die eigenthümlichen verzweigten Protoplasmazüge innerhalb der Fettkugel, welche zuerst von Carl Told.ti gesehen und abgebildet wurden. Das Präparat, aus welchem meine Abbildung stammt, ist von der Kopf haut eines an acuter Miliartuberculose verstorbenen Mannes, bei welchem die Abzehrung sehr rasch, binnen wenigen Wochen, stattgefunden hatte (Siehe Fig. 3).

$Z$

$F$

C

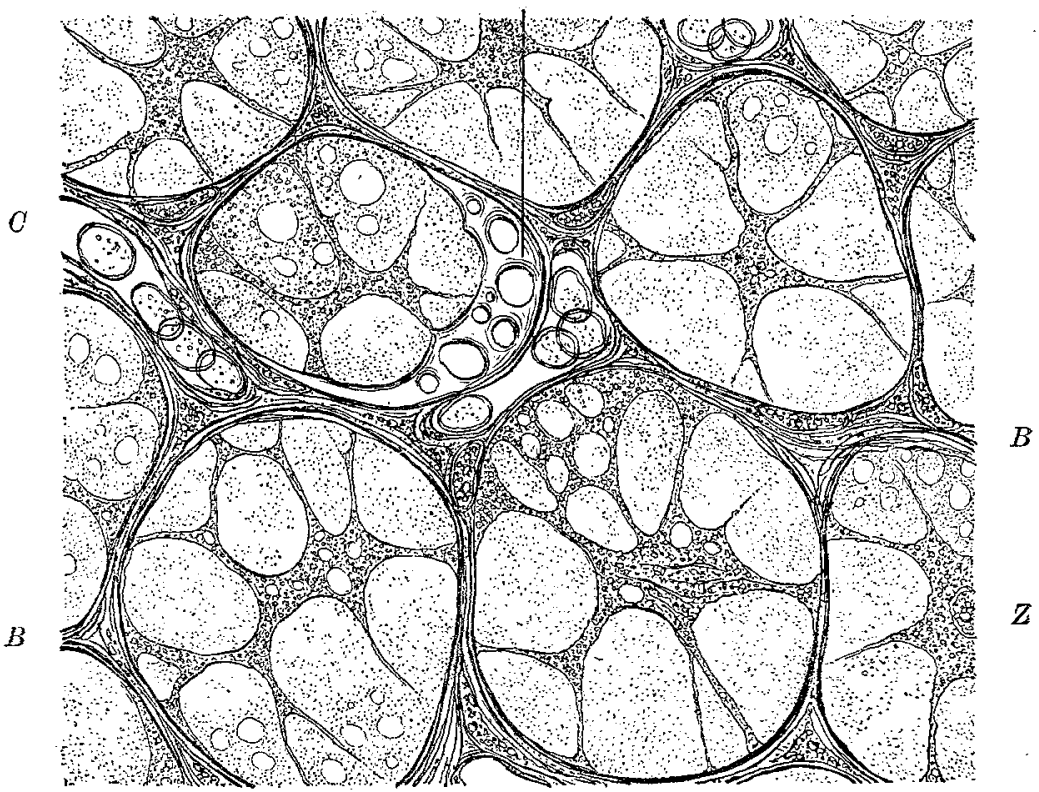

Fig. 3. Acuter Schwund des subcutanen Fettgewebes der Kopfhaut. Vergr. $=\mathbf{6 0 0}$.

$F$ Ueberrest von vacuolirtem Fett. $Z Z$ Verzweigte Protoplasmazüge nach Schwund des Fettes. $B B$ Bindgewebskapseln und die Fettkugeln. $C C$ Capillare Blutgefässe.

Wir sehen $a b$ und zu spärliche wandständige Ueberreste von durch Aufbewahrung in Glycerin vacuolirtem Fett. Alle Fettkugeln zeigen ein radiär verzweigtes, feingranulirtes Proto-

1) Lehrbuch der Gewebelehre. 3. Auflage 1888. 
plasmanetz, häufig von einem etwas grösseren centralen Protoplasmakörper ausgehend. In diesem Körper erkennen wir central gelegene grössere Körner, oder auch blasse kernartige Bildungen. In vielen Fällen trägt der centrale Körper eine wechselnde Anzahl von nadelförmigen, augenscheinlich postmortal aufgetretenen Krystallen von Margarinsäure. Die Maschenräume zwischen den verzweigten Aesten des Protoplasmas erscheinen sehr fein granulirt, und nur ab und $\mathrm{zu}$ gelingt es auch in den Maschenräumen die protoplasmatische Structur, reichlich von Vacuolen durchbrochen, zu erkennen.

Hier haben wir die unmittelbare Folge eines raschen Schwundes des Fettes vor uns. Wir sehen verzweigte Protoplasmakörper von grosser Aehnlichkeit mit jenen Körpern, die wir in einem Knochengewebsterritorium als Knochenkörperchen, im Faserknorpel als verzweigte Knorpelkörperchen und im myxomatösem Gewebe der Nabelschnur als Protoplasmanetz bezeichnen. Es sind dies Bildungen, welche sich innerhalb der Fettkugel nach erfolgter Infiltration mit der Fettgrundsubstanz gebildet haben, ebenso wie im Knochengewebe aus indifferentem Protoplasma die eigentlichen Knochenkörperchen zur Ansicht kommen, nach erfolgter Infiltration des Territoriums zuerst mit leimgebender Grundsubstanz und dann mit Kalksalzen. Dass die Maschenräume zwischen den Protoplasmazügen nicht völlig structurlos sind, können wir aus der ungemein zarten Körnung und der allerdings undeutlichen Protoplasmastructur der Felder der Grundsubstanz vermuthen.

Sicherheit erlangen wir über diesen Umstand durch das Studium des Unterhautfettgewebes von Individuen, bei welchen die Abzehrung allmälig erfolgt ist. Auch eignen sich hierzu Präparate der Haut aus der Umgebung chronischer Entzündungsprocesse, wie schon $\mathrm{C} z$ a j e w i c z gewusst hat. Das abgebildete Präparat stammt von der Brusthaut aus der Nähe eines eiternden Hohlganges, welcher zu einer durch Actinomycose hervorgerufenen necrotischen Rippe führte. (Siebe Fig. 4.)

Hier erkennen wir bei aufmerksamem Zusehen einen in jeder Fettkugel vorhandenen centralen Protoplasmakörper, welcher zahlreiche Fortsätze gegen die Peripherie der Fettkugel sendet. In den Maschenräumen zwischen den proto- 
plasmatischen Zügen treffen wir Protoplasmakörper ron beträchtlicher Grösse, von welchen einige offenbar in Folge der

$$
\text { K } \quad K \quad P
$$

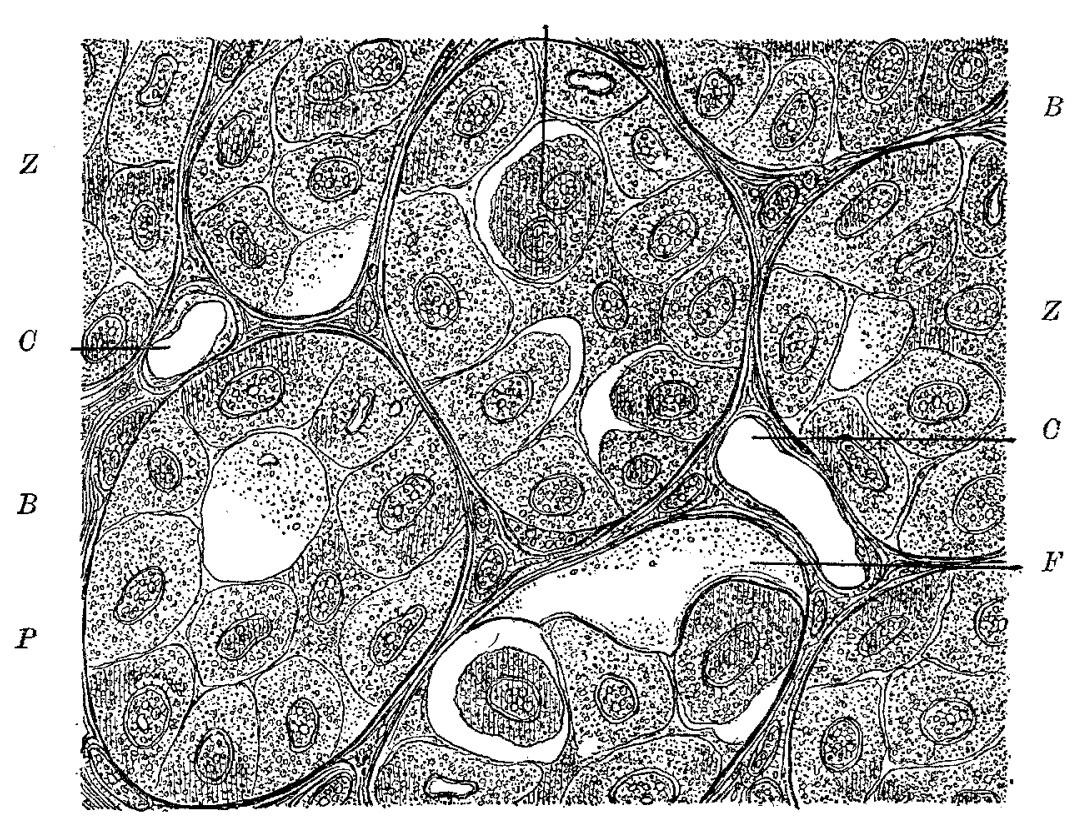

Fig. 4. Chroniseher Schwund des subcutanen Fettgewebes der Brusthaut. Vergr. $=600$.

$F^{\prime}$ Fein granulirter Ueberrest von Fets. $Z Z$ Verzweigte Protoplasmazüge. $P P$ Kernhaltige, feingranulirte Protoplasmakörper. $K K$ Protoplasmakörper mit je zwei Kernen. $B B$ Bindegewebskapseln um die ehemaligen Fettkrugeln. CC Capillare Blutgefässe.

Härtung in Chromsäurelösung sich von dem centralen Protoplasmakörper oder dessen Fortsätzen abgelöst haben. Sowohl der centrale, wie auch die peripherischen Körper bestehen aus sogenanntem blassem oder feinkörnigem Protoplasma. Die meisten der peripheren Körper enthalten je einen grossen, blasenförmigen oder einen kleinen, soliden, unregelmässigen Kern. Manche dieser Körper enthalten zwei Kerne. Hie und da fehlt der Protoplasmakörper und wird durch eine feinkörnige Masse ersetzt, die schon von Toldt richtig als schleimige Grundsubstanz erkannt worden ist. Das interstitielle Bindegewebe ist 
Normale und patholog. Histologie des Unterhaurfettgewebes. 357

mit einer normalen Anzahl von Kernen versehen, nirgends verbreitert, so zwar, dass ein Entzündungsprocess hier nicht mit Sicherheit nachgewiesen werden kann. Augenscheinlich haben wir hier ein Bild vor uns, wie es schon ron Fle $\mathrm{mm}$ ing gesehen und mit dem eigenthümlichen Ausdrucke "Wucheratrophie" bezeichnet wurde. Es kann wohl keinem Zweifel unterliegen, dass es sich hier weder um einen Wucherungsnoch um einen atrophischen Process handelt, sondern einfach um eine Rückkehr zum embryonalen Zustande der Fettkugel nach Schwund des Fettes. Die doppelten Kerne mancher Protoplasmakörper weisen nicht auf Wucherung hin, wie Flem ming geglaubt hat, sondern auf eine fortschreitende Abspaltung zu kleineren Protoplasmakörpern, entsprechend den wechselnden Grössen jener Körper, aus welchen sich ursprünglich die spätere Fettkugel gebildet hat.

Aus dem Studium solcher Bilder wird sofort klar, dass die Fettkugel keineswegs eine einzige und einfache Zelle darstellen kann. Sie ist im Gegentheile das Product einer ganzen Anzahl ron Protoplasmakörpern, gerade so, wie ein Feld der myxomatösen Grundsubstanz, in welcher sich ein centraler kernhaltiger Körper erhalten hat (Placenta), oder in welcher eine Anzahl kernartiger Körper zurückgeblieben sind ( $\mathrm{Lymph-}$ gewebe). Obgleich sämmtliche Protoplasmakörper der Fettkugel fein granulirt sind, lässt sich der netzförmige Bau. dieser Körper bei stärkeren Vergrösserungen leicht erkennen. Von den Kernkörperchen ziehen radiäre Speichen zu den Nachbarn sowohl, wie zur Kernschale. Aus der letzteren ziehen wieder zarte Speichen zu den nächsten Körnchen und sämmatliche Körnchen sind untereinander durch Fädchen verbunden, welche sich auch in den centralen Protoplasmakörper und dessen Fortsätze einsenken. Das Netz der lebenden Materie, wie es von meinem Vater bezeichnet wurde, ist nirgends unterbrochen. Offenbar sind es die Maschenräume dieses Netzwerkes, welche im Knorpelgewebe mit chondrogener, im Knochengewebe mit leimgebender, im myxomatösen Gewebe mit mucinhaltiger, und im Fettgewebe mit einer Fettgrundsubstanz infiltrirt sind. Unter allen Umständen bleibt das Netz der lebenden Materie innerhalb der Grundsubstanz erhalten, obgleich dasselbe auf der 
Höhe der Infiltration mit Grundsubstanz unsichtbar geworden ist.

Ueber die Herkunft des Fettes können wir mit Bestimmtheit aussagen, dass es ein Umwandlungsproduct der lebenden Materie ist. In den Epithelien der Brustdrüse, kurze Zeit nach erfolgter Entbindung, begegnen wir Fettkügelehen, die sich mittelst Osmiumsäure schwarz färben lassen, und die noch mittelst zarter Fädchen mit dem umgebenden Netze der lebenden Materie in Verbindung stehen. Dasselbe ist an Colostrumkörperchen, den Abkömmlingen der Brustdrüsenepithelien, der Fall. S. S t r i c k e r hat schon längst die Beobachtung gemacht, dass am geheizten Objectträger die Fettkörnchen aus den Colostrumkörperchen ausgepresst werden. Auf der Höhe der Lactation wird der grösste Theil der lebenden Materie der Brustdrüsenepithelien sehr schnell zu Fettkörnchen umgewandelt, welche aus den Epithelien entleert, die Milchkïgelchen liefern. Allerdings erfolgt auch rasche Neubildung der lebenden Materie als Ersatz der durch Milchbildung verloren gegangenen. Im subcutanen Fettgewebe ist der Process derselbe, nur wird stets ein Antheil der Knotenpunkte der lebenden Materie zu Fett umgewandelt, während ein zartes Netz zurückbleibt, um die halbflüssige Substanz, die jetzt die Rolle von Grundsubstanz übernommen hat, zu durchziehen.

Die Entwickelungsgeschichte des Fettgewebes liefert den Beweis, dass die Fettkugeln aus Protoplasmakörpern hervorgehen. (Siehe Fig. 5.)

Das Präparat war das eines senkrechten Schnittes der Haut der seitlichen Halsgegend eines menschlichen Foetus in der ersten Hälfte des fünften Schwangerschaftsmonates. Fettläppchen, insbesondere grössere, sind um diese Zeit noch spärlich. Ueber die Structur der Fettkugeln liefert uns das Präparat keinen Aufschluss, weil dasselbe mit Alkohol und Aether behufs Einbettung in Celloidin behandelt worden war. In Folge davon enthalten die Fettkugeln nur spärliche Ueberreste von Fett. Hie und da sieht man verzweigte blasskörnige Protoplasmakörper, aber so verstümmelt, dass sie uns keine richtige Vorstellung über das das l'ett durchziehende Protoplasmanetz gestatten. Zwischen den schön geformten Fett- 
kugeln sehen wir zahlreiche kernhaltige Protoplasmakörper von wechselnder Grösse. Viele davon tragen Vacuolen, wahrscheinlich früher mit Fettröpfchen erfüllte Hohlräume. Es kann keinem Zweifel unterliegen, dass sich je eine Fettkugel aus einer Anzahl solcher indifferenter Protoplasmakörper entwickelt. Ich möchte betonen, dass eine Anzahl dieser Körper die Fettkugel herstellt, im Widerspruch mit anderen Autoren, die sich

$F$

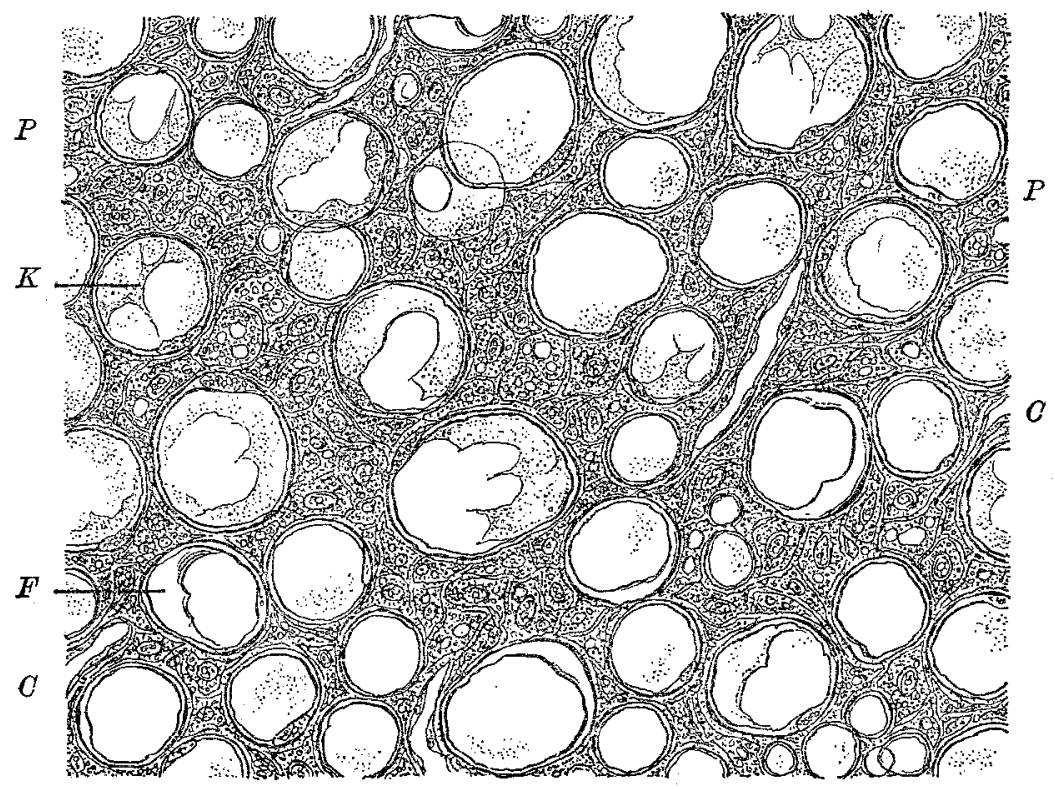

Fig. 5. Aus einem Fetträubchen der Haut eines 5monatlichen menschlichen Foetus. Vergr. $=600$.

$F F$ Ueberreste von Fett. $K K$ Fettkugeln mit verzweigten Protoplasmazügen. $P P$ Indifferente Protoplasmakörper, zum Theile vacuolirt. $C C \mathrm{Ca}$ pillare Blutgefässe.

der Vorstellung hingeben, dass je ein Protoplasmakörper zu einer Fettzelle wird. Mit dieser Auffassung lassen sich die Abzehrungsbilder der Fettkugel (siehe Fig. 4) ungezwungen erklären.

Protoplasmakörper, die man seit Wald e y e r und E h r li ch als "grobgranulirte" oder "Mastzellen" kennt (siehe Fig. 2, M.), sind höchst wahrscheinlich die Vorläufer der Fettkugelbildung. 
Die groben, ziemlich stark glänzenden Körner solcher Bildungen geben nicht mehr die Reaction von Proteinaten, aber auch die Reaction der Osmiumsäure ist noch nicht ausgesprochen. Die genannten Autoren haben übersehen, dass die Körnchen nicht isolirte Bildungen sind, sondern mittelst feiner Fädchen mit den Nachbarbildungen im Zusammenhange stehen. In diesen Körpern werden die Knotenpunkte des das Protoplasma durchzieherden Netzes auf eine chemisch bisher nicht aufgeklärte Weise zu Fettkörnern umgewandelt. Eine Fettkugel kann eine einzelne, sogenannte Mastzelle nicht liefern. Es ist indessen recht wohl möglich, dass durch die Mastzellen die Bildung von Fettkugeln eingeleitet wird, in ähnlicher Weise, wie bei dem Ossificationsprocesse häufig eine Kalkablagerung in einzelnen Protoplasmakörpern vorausgeht.

Von besonderem Interesse sind die En tzünd ung s bilder des subcutanen Fettgewebes. Die Frage, ob aus den Fettkugeln direct Entzündungs- resp. Eiterkörper hervorgehen, ist neuepdings durch die Arbeiten aus der Schule von P. Grawitz in Greifswald in Fluss gekommen. H. Schmidt stellt in seiner Arbeit „Schlummernde Zellen im normalen und pathologisch veränderten Fettgewebe" ${ }^{1}$ ) die Behauptung auf, dass die normale, sogenannte Fettzelle ein einheitliches Gebilde sei, herrorgegangen aus einer einzigen Zelle. Ferner behauptet er, dass die Fettkugel, oder wie er es nennt, der fertige ruhende Fettbehälter als eine Vielheit von Zellen, als ein Zellrerband anzusehen sei, dessen einzelne Componenten in der Membran desselben in einer für uns unsichtbaren Form vorhanden sind - Schlummerzellen. Bei gewissen Reizzustönden löst sich nach ihm der Zellverband wieder in seine einzelnen Elemente auf, $d . h$. die vorher anscheinend aus einer homogenen Faser bestehende Membran zeigt zunächst Kerne, welche weiterhin zu Zellen werden. Aus letzteren Zellen entwickelt sich ein gefässreiches Granulationsgewebe.

Ich kann mich der Auffassung von Schmidt über die Entstehung von Entzündungskörpern aus Schlummerzellen nicht anschliessen. Für mich gibt es weder im Fett- noch in irgend einem anderen Gewebe Zellen, und noch weniger Schlummer-

I) Virchow's Archiv. Band 128, 1892. 
zellen. Da sämmtliche Protoplasmakörper der Gewebe untereinander in Verbindung stehen, dürfte die Vorstellung von individuellen Zellen hinfällig sein. „Schlummerzellen" kann ich deshalb nicht anerkennen, weil nicht nur das Protoplasma, sondern auch sämmtliche Grundsubstanzen, folglich auch das Fett, ron einem zarten Netze lebender Materie durchzogen wird. Der letztere Antheil schlummert nicht, sondern ist nur in der Grundsubstanz verborgen. Sobald letztere verflïssigt wird und schwindet, kommen die Protoplasmakörper zur Ansicht, welche ehedem die Grundsubstanz aufgebaut haben. Von dem lebenden Antheile dieser Körper gehen durch Anwachsen neue Elemente hervor, die bei der Entzündung entweder neue Gewebe produciren oder zu Eiterkörperchen zerfallen.

Ich habe für die Darstellung der formativen oder plastischen Entzündung des Fettgewebes eine Stelle der Brusthaut von dem oben erwähnten Falle von Actinomycose mit Necrose einer Rippe gewählt, in grösserer Nähe des eiternden Hohlganges. (Siehe Fig. 6.)

In den Fettkugeln sehen wir Bilder, die stark an die Abzehrungsbilder (Fig. 4) erinnern. Viele Protoplasmakörper sind in Folge der Entziindung grobgranulirt geworden, und mit homogenen aus solider lebender Materie bestehenden Kernen versehen. Im Gegensatze zu diesen grobgranulirten Protoplasmakörpern begegnen wir solchen, deren Protoplasma und Kerne im Abblassen, demnach in Umwandlung zu schleimiger Grundsubstanz begriffen sind. Da das Balkenwerk zwischen den Protoplasmakörpern deutlich sichtbar ist, an den Knotenpunkten sogar nengebildete Kerne aufweist, ist das Bild eines aus der Fettkugel hervorgegangenen myxomatösen Granulationsgewebes recht augenfällig. Manche Protoplasmakörper der ehemaligen Fettkugel sind zu kleineren Elementen zerfallen (Fig. 6 E); das sind Entzündungskörper, welche das myxofibröse interstitielle Gewebe verstärken. Bei vorgeschrittener Bildung von myxomatösem und myxofibrösem Granulationsgewebe geht die Form der ehemaligen Fettkugel ganz zu Grunde und nur durch grosse, in das myxofibröse Gewebe eingestreute Protoplasmakörper, werden wir an das ehemalige Fettgewebe gemahnt. 
Die aus den Protoplasmakörpern der ehemaligen Fettkugel hervorgegangenen Entzündungskörper können auch, anstatt
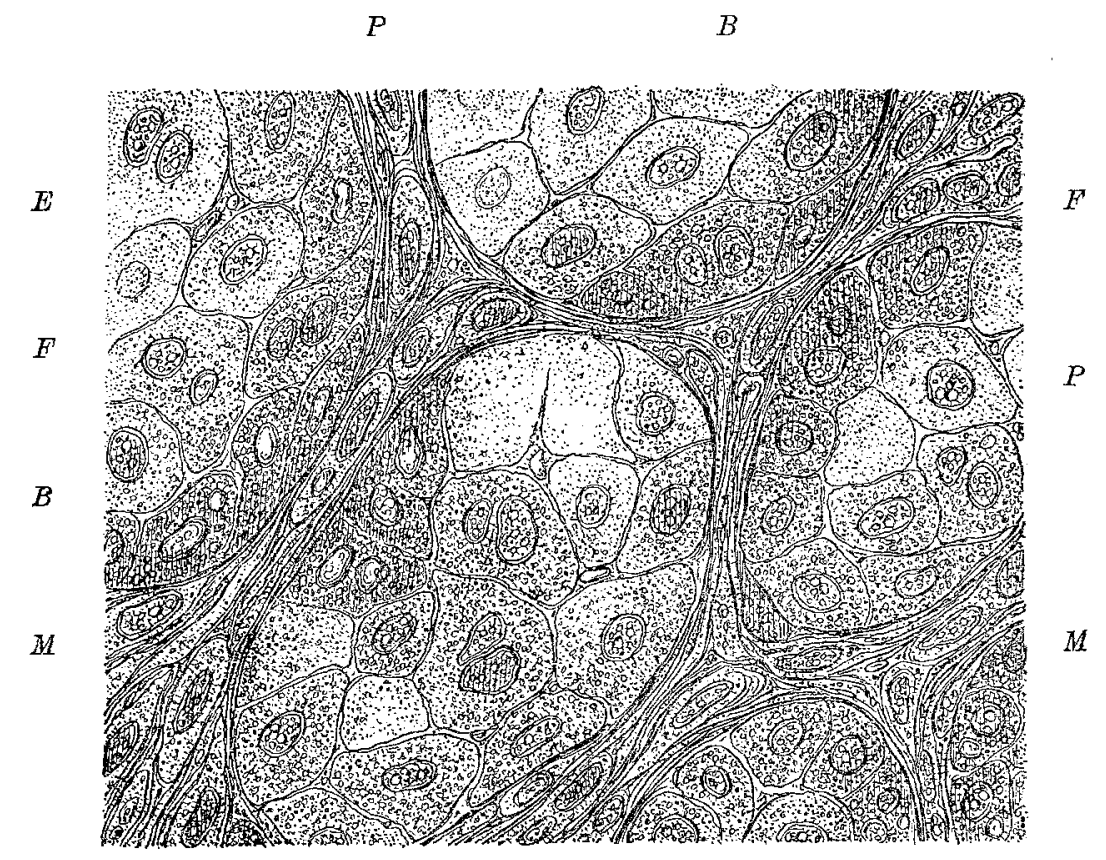

Fig. 6. Umwandlung des Fettgewebes zu myxomatösem Granulations-Gewebe. Vergr. $=600$.

PP Protoplasmakörper mit grobgranulirten oder homogenen Kernen. $E$ Entzündungskörper aus der Fettkugel hervorgegangen. $M M$ Wiederauftreten von myxomatöser Grundsubstanz. $F F^{\prime}$ Interstitielles myxo-fibröses Gewebe. $B B$ Balkenwerk des myxomatösen Gewebes.

ein neues Gewebe bilden zu helfen, aus dem gegenseitigen Verbande gerissen und zu Eiterkörperchen umgewandelt werden. Ich habe diesen Vorgang bei Eiterungen des cutanen und subcutanen Gewebes, insbesondere bei eiteriger Mastitis beobachtet, mit völligem Zugrundegehen der Eiterkugeln.

Nicht nur im Entzündungsprocesse, sondern auch bei Geschwulstbildung betheiligen sich die FettkugeIn in activer Weise, selbstrerständlich nach erfolgter Auslösung des Fettes und Wiederauftreten der Protoplasmakörper, welche die Fett- 
Normale und patholog. Histologie des Unterhautfettgewebes. 363

kugel ursprünglich aufgebaut haben. Dieser Vorgang ist insbesondere bei der Wucherung des Rundzellen-Sarcoms schön zu verfolgen, und hoffe ich darauf bei anderer Gelegenheit zurückzukommen.

Die Ergebnisse meiner Studien lassen sich in folgenden Punkten zusammenfassen:

1. Die Fettkugel ist keine Zelle, sondern aus einer grossen Anzahl indifferenter Protoplasmakörper aufgebaut. Der Kern hat mit der Fettkugel nichts zu schaffen, sondern liegt stets in der die Fettkugel einhüllenden Kapsel.

2. Die Kapsel der Fettkugel ist als eine elastische Schicht leimgebender Grundsubstanz zu betrachten, analog den an den Grenzen der Territorien des Knochen- und Knorpelgewebes auftretenden elastischen Schichten.

3. Das Fettgewebe ist als eine Varietät von myxomatösem Gewebe aufzufassen, ähnlich dem Lymphgewebe. In der Fettkugel bildet sich ein verzweigter Protoplasmakörper aus, ähnlich den verzweigten Protoplasmakörpern des Faserknorpels und des Knochens.

4. Der verzweigte Protoplasmakörper kann durch Reagentien nicht zur Anschauung gebracht werden, wird aber sofort sichtbar nach raschem Verschwinden des Fettes bei rapider Abmagerung.

5. Bei allmäliger Abmagerung werden in der Fettkugel nebst dem rerzweigten Protoplasmakörper auch solche kernhaltige Körper sichtbar, welche die Maschenräume des verzweigten Körpers ausfüllen. Keiner dieser Körper ist neu entstanden, sondern nur nach Auslösung des Fettes sichtbar geworden.

6. Die die Maschenräume erfüllenden Protoplasmakörper sind identisch mit jenen, aus welchen die Fettkugel bei ihrer Entwickelung aufgebaut worden ist.

7. Fettlkugeln sind nicht immer zu einem Gewebe vereinigt, sondern treten auch isolirt oder in kleinen Gruppen innerhalb des fibrösen Bindegewebes auf.

8. Die im fibrösen Bindegewebe häufig sichtbaren sogenannten grobkörnigen oder Mastzellen stellen höchst wahrscheinlich den Uebergang von Protoplasma zu Fett dar, indem 
die Knotenpunkte des Netzwerkes der lebenden Materie unmittelbar zu Fett umgewandelt werden.

9. Im Entzündungsprocesse werden die die Fettkugeln aufbauenden Protoplasmakörper durch Proliferation shrer lebenden Materie zu Entzündungskörpern umgewandelt, aus welchen bei der formativen Entzündung myxomatöses und myxofibröses Gewebe entsteht.

10. Wenn die aus der Fettkugel hervorgegangenen Entzündungskörper aus dem gegenseitigen Zusammenhange gerissen werden, entsteht aus der ehemaligen Fettkugel ein Haufen von Eiterkörpern. 